\title{
Small Cell Carcinoma of the Cervix: About Two Cases and Literature Review
}

\author{
Bouziane $A^{1 *}$, Abboud $F^{1}$, Ait Erraisse $\mathbf{M}^{1}$, Youssoufi Moulay $A^{2}$, \\ Astaoui $\mathrm{F}^{3}$, Farhan FZ1 ${ }^{1}$, Alami Z ${ }^{1}$, Bouhafa $\mathrm{T}^{1}$ and Hassouni $\mathrm{K}^{1}$ \\ ${ }^{1}$ Department of Radiation Oncology, University hospital Hassan II, Morocco \\ ${ }^{2}$ Medical Physics Unit, Oncology hospital, University hospital Hassan II, Morocco \\ ${ }^{3}$ Department of Hematology and Pediatric Oncology, University hospital Hassan II, \\ Morocco
}

\section{Research Article}

Volume 2 Issue 2

Received Date: December 03, 2019

Published Date: December 13, 2019

DOI: $10.23880 /$ ijnmrs-16000119

*Corresponding author: Bouziane Amina, Department of Radiation Oncology, University hospital Hassan II, Morocco, Tel: 0644031209; Email: amina.amina.bzn@gmail.com

\section{Abstract}

Neuroendocrine carcinomas with small gynecological cells are unusual, given their rarity and the absence of randomized trials, the diagnostic and therapeutic management of these tumors is difficult and is essentially modeled on that of pulmonary neuroendocrine tumors.

Methods: We report two new cases of small cell cervical neuroendocrine carcinoma and through the literature data we review the different aspects of this rare entity, both patients had a tumor stage IB2 and IIIC, the first The patient received RCC, followed by surgery, then postoperative brachytherapy, supplemented with adjuvant chemotherapy, and the second received RCC followed by brachytherapy.

Results: Both patients died within 6 to 18 months after the end of chemoradiotherapy following metastatic recurrence.

Conclusion: Despite multimodal treatment, the prognosis of neuroendocrine carcinomas remains unfavorable.

Keywords: Small Cell Neuroendocrine Carcinoma; Cervix; Radiation Therapy; Chemotherapy

Abbreviations: SCCC: Small Cell Carcinoma of the Cervix; SCLC: Small Cell Lung Carcinoma; H\&E: Hematoxylin and Eosin; MRI: Magnetic Resonance Imaging; FFS: Failure-Free Survival; TGOG: Taiwanese Gynecologic Oncology Group; CSS: Cancer-Specific Survival; FIGO: International Federation of Obstetrics and Gynecology.

\section{Introduction}

Small cell carcinoma of the cervix (SCCC) is a rare neuroendocrine malignancy comprising less than $3-5 \%$ of cervical carcinomas [1]. SCCC is a high-grade neuroendocrine tumor that shares numerous characteristics of small cell lung carcinoma (SCLC) and has often been considered an "extrapulmonary" small cell carcinoma [2,3]. Classic neuroendocrine morphological characteristics of SCCC and SCLC include small "blue" cells with hyperchromatic nuclei and scant cytoplasm on hematoxylin and eosin (H\&E) staining, as well as frequent mitotic figures with inconspicuous or absent nucleoli. Along with shared morphological characteristics and architectural patterns, SCCC and SCLC share many immunohistochemistry markers, such as synaptophysin, 
chromogranin A, and CD56 [4]. The diagnosis of primary SCCC is made by cervical biopsy noting histopathologic features characteristic of small cell carcinoma, along with exclusion of primary SCLC (3). While SCCC bears similar histopathologic features and clinical behavior to SCLC and has historically been considered an extrapulmonary variant of the same malignancy, recent advances in molecular and genetic research have indicated that small cell carcinomas in different anatomic sites carry distinct genetic markers and are actually unique diseases [5].

SCCC is a very aggressive malignancy with frequent lymph node involvement at the time of diagnosis (1). Most patients treated for SCCC experience treatment failure secondary to early local recurrence as well as distant metastases, which occur most commonly in the lung, liver, brain and bone [6]. The combined survival rate reported for all stages of SCCC ranges from 11 to 54\%, with the overall prognosis being poor for advanced disease [6].

\section{Materials and Methods}

\section{Observation 1}

Ms. BK, age 62, 4th gesture 4th parity, menopausal at the age of 48, patient had no history of HPV infection, or other sexually transmitted infection, first intercourse was at the age of 24; The onset of her illness dates back to a month before her consultation with post-coital metrorrhagia of low abundance, without digestive or urinary signs. There was no notion of alteration of the general state. The clinical examination found a patient in good general condition. The gynecological examination revealed a budding process in the posterior lip, $1.5 \mathrm{~cm}$ long, bleeding on contact. At the pelvic touches the uterus was slightly enlarged and the parameters were free. The patient underwent a biopsy of the cervical budding process, and the histological study revealed a poorly differentiated and infiltrating carcinoma of the cervix. Immunolabelling found small cell neuroendocrine carcinoma with positivity of anti-Pan-cytokeratin antibodies, anti-CK7 antibodies and anti-chromogranin antibodies.

Pelvic magnetic resonance imaging (MRI) showed a cervical tumor process measuring $20 \mathrm{~mm}$ long axis, without extension to parameters, vaginal walls and uterus, and without pelvic lymphadenopathy (Figure 1). The patient had a thoracoabdominopelvic CT scan (CT TAP); as part of the extension assessment, which had not shown secondary location. The disease has been classified according to the classification of the International Federation of Obstetrics and Gynecology 2018 (FIGO 2018) stage IB2.

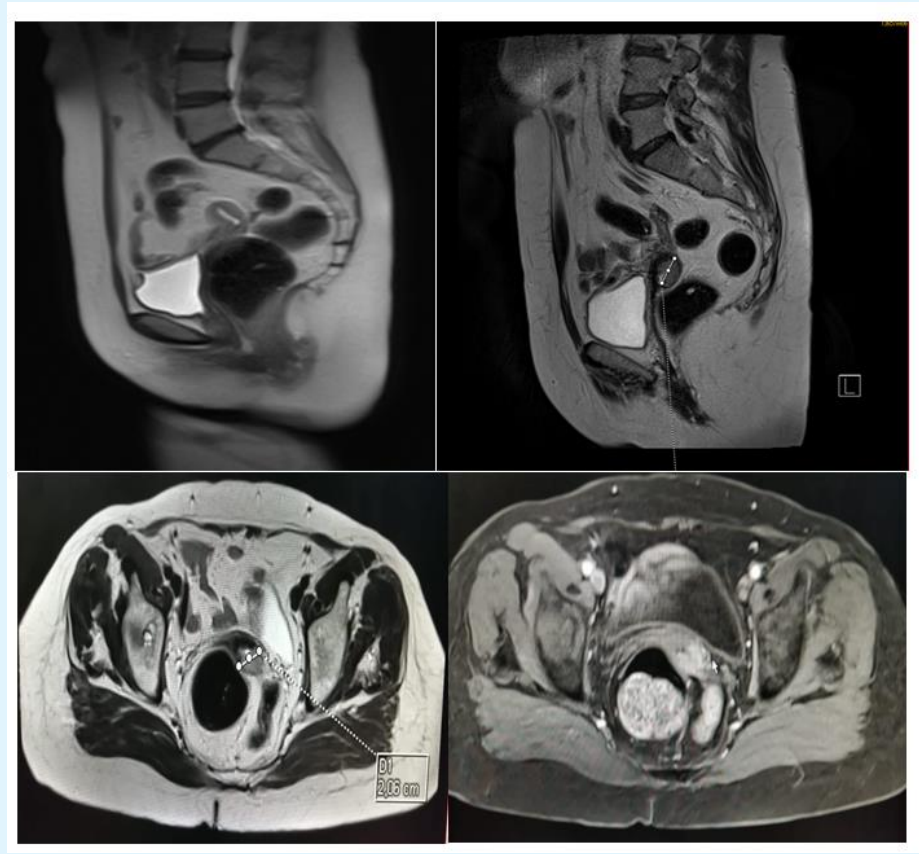

Figure 1: Sagittal and axial section of pelvic MRI in sequence T2, T1 FAT-SAT with contrast injection showing a tumor process on the right posterolateral side of the cervix, stage IB2 according to FIGO 2018 classification. 


\section{International Journal of Nuclear Medicine \& Radioactive Substances}

The indication selected in a multidisciplinary consultation meeting (RCP) was concomitant radio chemotherapy, External radiotherapy was delivered by four X-ray beams of $18 \mathrm{MV}$ in the so-called «boxed » technique, in an isocentric manner. The upper limit of the irradiated volume was on line L5 -S1 (Figures 2a \& 2b). The total dose delivered to the isocenter was 46 Gy in 23 fractions of $2 \mathrm{~Gy}$, one fraction per day and five fractions per week, spread over a 27-day period.
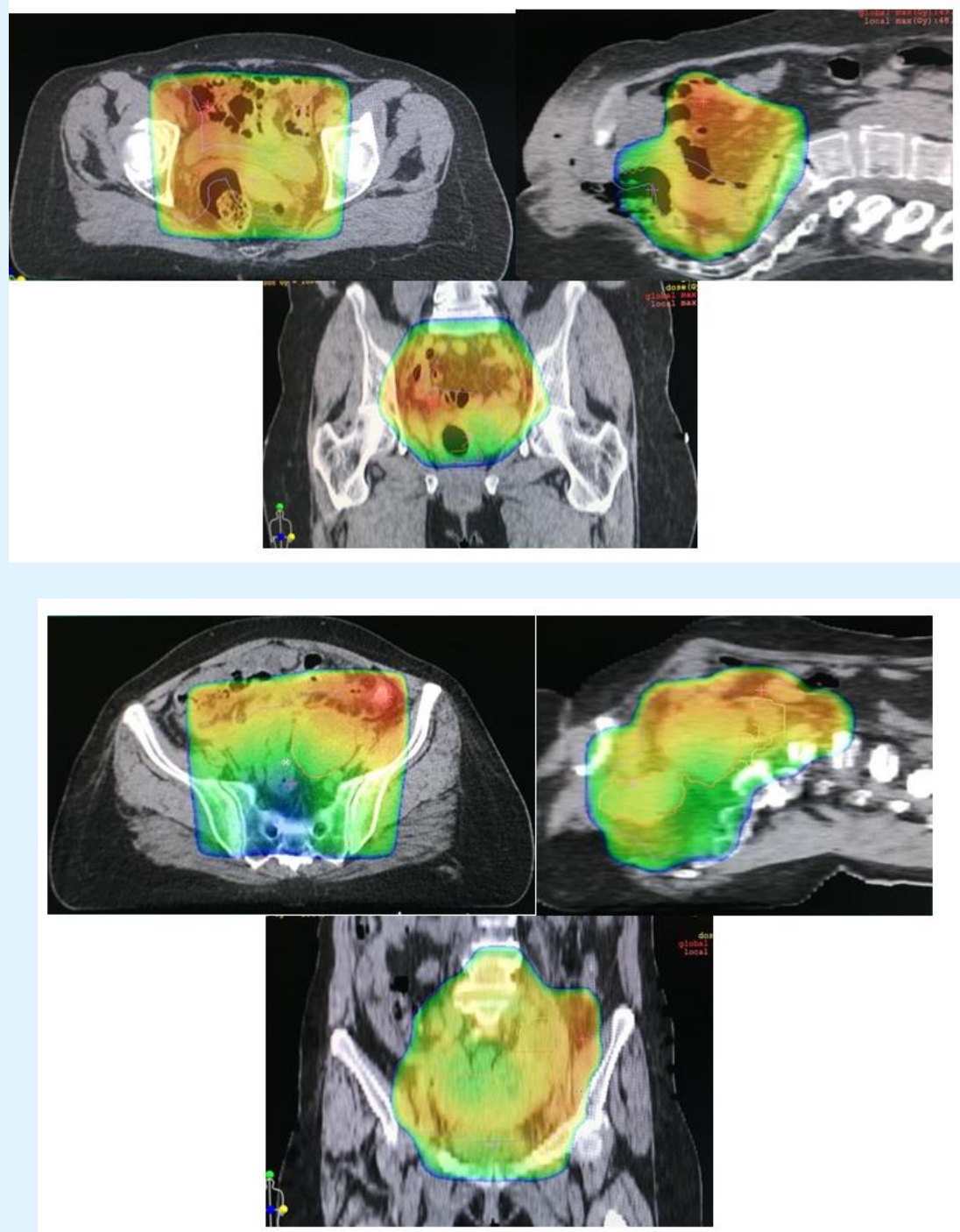

Figure 2a \& 2b: Scanning sections showing dose distribution during external radiotherapy.

External radiotherapy was followed by high dose rate utero-vaginal brachytherapy using a fletcher applicator loaded with 24 Gy sources of iridium 192 (Figures 3a \& 3b).

The patient received concomitant chemotherapy with cisplatin $80 \mathrm{mg} / \mathrm{m} 2$ on day 1 combined with etoposide
$100 \mathrm{mg} / \mathrm{m} 2$ from day 1 to day 3, three courses of treatment (one course every 3 weeks). The patient's file was restored to multidisciplinary consultation meeting (RCP) and the indication of surgery was retained. Indeed, the patient benefited from an enlarged colpohysterectomy with ilio-obturator lymphadenectomy. The histological study of the hysterectomy specimen 
confirmed the diagnosis of undifferentiated small cell neuroendocrine carcinoma, the parameters are free from any tumor invasion, with no ganglionic involvement.

The patient underwent post-operative high dose rate brachytherapy, on the vaginal slice 10 Gy in 2 fractions, by miami-type applicator loaded with sources of iridium 192 (Figure 3). Followed by adjuvant chemotherapy with cispaltine combined with etoposide five cures (one course every 3 weeks).
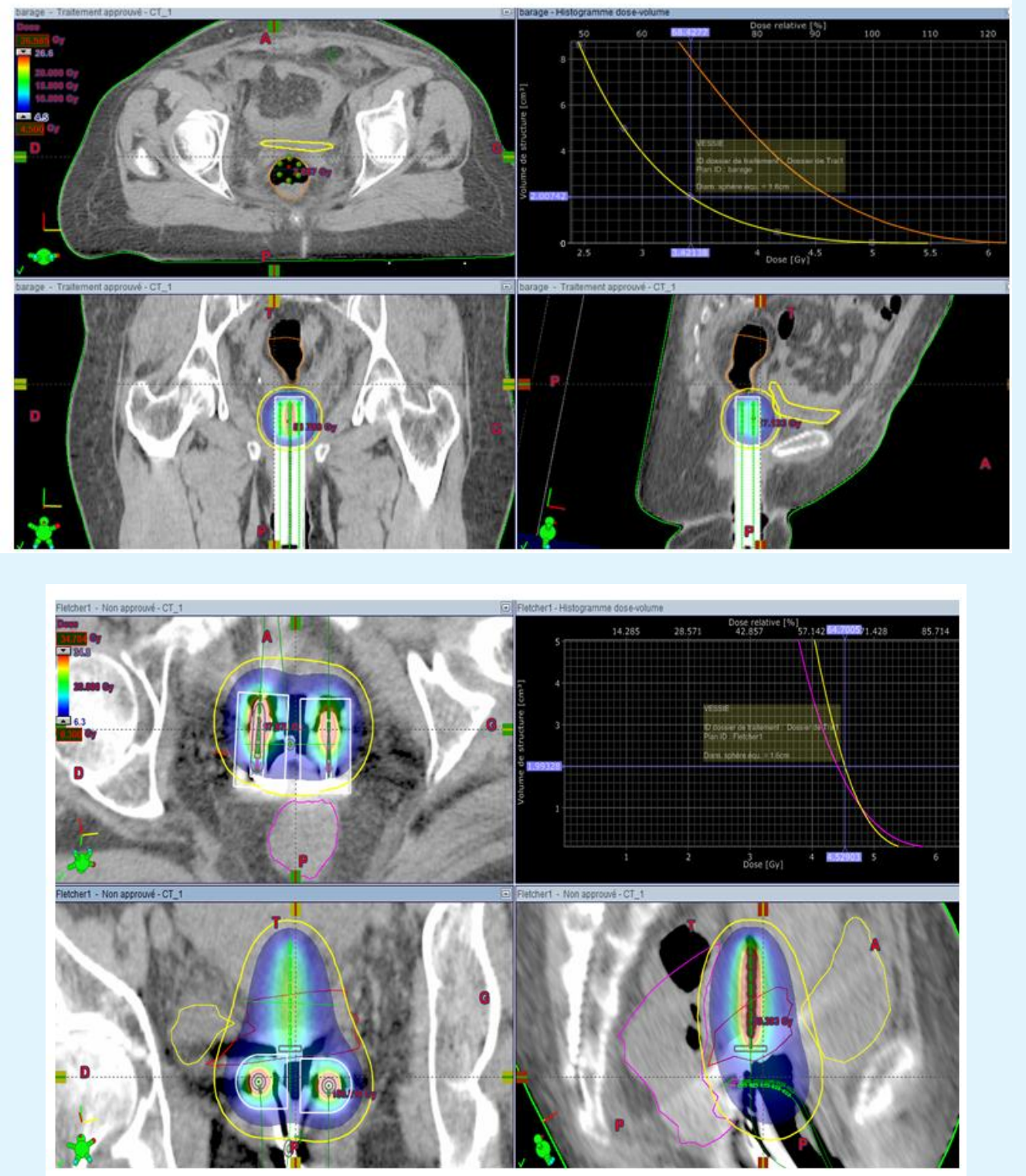

Figures 3a \& 3b: Scanning sections showing dose distribution during brachytherapy and dose-volume histogram of at-risk organs (bladder and rectum).

The patient received concomitant chemotherapy with cisplatin $80 \mathrm{mg} / \mathrm{m}^{2}$ on day 1 combined with etoposide
$100 \mathrm{mg} / \mathrm{m}^{2}$ from day 1 to day 3 , five courses (one course every 3 weeks). 


\section{International Journal of Nuclear Medicine \& Radioactive Substances}

\section{Observation 2}

Ms. SB, age 59, 7th gesture 7th parity, menopausal at the age of 54, patient had no history of HPV infection, or other sexually transmitted infection, first intercourse was at the age of 16; The first alarming clinical sign was metrorrhagia of low abundance with dyspareunia, without digestive or urinary signs all evolving in a context of conservation of the general state.

The gynecological examination revealed a hard ulcerobudding process of the cervix measuring $6 \mathrm{~cm}$ and invading the upper third of the vagina. At the pelvic touches the uterus was slightly enlarged and the distal parameters were infiltrated. The patient underwent a biopsy of the ulcero-budding process of the cervix and the histological study concluded that there was tumor proliferation made of small nuclei and more or less thick papillary structures; the cells are of medium size with rounded or ovoid hyperchromatic nucleus with moderate anisocaryosis and some mitotic figures.

The cytoplasm is basophilic without mucosecretion. The complementary immunohistochemical study carried out on paraffin-embedded sections shows an intense labeling of the carcinomatous cells using the antichromogranin A antibody. Some cells are discretely labeled with the CD56 antibody. Thus the cytoarchitectural and immunohistochemical appearance was compatible with small cell carcinoma of the cervix. Pelvic magnetic resonance imaging found a voluminous lesion of the cervical outer margin with left parametrial involvement, associated with right external iliac lymph node involvement, with the presence of a large gangliotumor mass. The patient had a thoracoabdominopelvic CT-scan (CT TAP); as part of the extension assessment, which had not shown secondary location.

The disease has been classified according to the classification of the International Federation of Obstetrics and Gynecology 2018 (FIGO 2018) stage IIIc. The indication chosen in a multidisciplinary consultation meeting (RCP) was concomitant radio chemotherapy. External radiotherapy was delivered by four X-ray beams of $18 \mathrm{MV}$ in the so-called "boxed " technique, in an isocentric manner. The upper limit of the irradiated volume was on line L4-L5 (Figure 4), The total dose delivered to the isocentre was 46 Gy in 23 fractions of 2 Gy, a complement of 10 Gy was delivered on the parameters and the right external iliac lymphadenopathy, one fraction per day and five fraction per week, spread over a period of 44 days.

\section{Results}

\section{Observation 1}

The clinical examination of the patient after one month of irradiation did not find signs of local or distant recurrence, a thoraco-abdominopelvic CT-scan was performed at 2 months after the end of irradiation which showed complete remission. The patient remained in good control loco regional and remote for 14 months. Subsequently, the patient presented herself in consultation for management of general deterioration with an increase in the volume of the lower limbs. The clinical examination found a patient with OMS at 2, the gynecological examination found a synecic vagina at the top two-thirds, the ganglionic areas were free. At general examination there was edema of the two lower limbs, taking the scoop, without inflammatory signs opposite. The TAP CT-Scan showed a regional recurrence peritoneal carcinomatosis type. The patient refused any kind of treatment and she died after 18 months of the end of treatment.

\section{Observation 2}

The clinical examination of the patient after one month of irradiation did not find signs of local recurrence or remotely, a thoraco-abdominopelvic computed tomography was performed at two months after the end of irradiation which showed a Cervical-centered tissue process of $31 * 29 \mathrm{~mm}$ with fluid retention upstream. The patient was referred for closing hysterectomy but was rejected by the gynecologist. Subsequently the patient presented in consultation after four months for management of abdominal distension with an increase in volume of the lower left limb.

Clinical examination found a patient with OMS at 2, distended abdomen, gynecological examination found a synovial vagina at the top two-thirds, and at rectal scan buttock arch of Douglas, ganglionic areas were free. On general examination there was edema of the left lower limb taking the scoop and no inflammatory signs. The venous Doppler echo of the lower limbs showed deep vein thrombosis; for which the patient was put on anticoagulant treatment, the TAP CT-Scan objectified a recurrence peritoneal carcinosis, with pulmonary metastases. The patient was placed on chemotherapy palliative carboplatin type AUC 4 etoposide, she received a single cure, then the patient died after 06 months of the end of treatment. 


\section{International Journal of Nuclear Medicine \& Radioactive Substances}

\section{Discussion}

The study analyzing locally advanced SCCC is a retrospective review by Wang et al. that examined 179 patients who underwent primary treatment of FIGO stages I-IV SCCC between 1987 and 2009 at member hospitals of the Taiwanese Gynecologic Oncology Group (TGOG) [7]. Of the patients with stages IIB-IVB disease (n = 56), any primary treatment with EP for at least five cycles was associated with significantly better 5-year failure-free survival (FFS) $(42.9 \%$ vs. $11.8 \%, \mathrm{p}=0.041)$ and cancer-specific survival (CSS) $(45.6 \%$ vs, $17.1 \%$, p = $0.035)$ relative to other treatments (7). Additionally, Wang et al. demonstrated that CCRT utilizing at least five cycles of EP was associated with a markedly improved FFS $(62.5 \%$ versus $13.1 \%, \mathrm{p}=0.025)$ and 5 -year CSS $(75.0 \%$ versus $16.9 \%, \mathrm{p}=0.016)$ compared to other treatments (7). This TGOG study additionally underscored the superiority of CCRT over chemotherapy alone.

While SCCC has historically been regarded an extrapulmonary variant of SCLC, recent advances have indicated that small cell carcinomas in different anatomic sites carry distinct genetic markers and are unique diseases (5). Consequently, although the chemotherapy agents utilized for SCCC have been chosen based on studies conducted on SCLC in the absence of phase II or phase III data [8], local treatment with RT for this malignancy should emulate local treatment for nonneuroendocrine cervical carcinomas as opposed to the RT regimen utilized for SCLC. An analysis of the National Cancer Database studying patients with locally advanced SCCC found that brachytherapy is an integral aspect of definitive CRT for SCCC and should not be omitted [9]. Of the 100 patients with locally advanced SCCC analyzed, the addition of brachytherapy was associated with a median survival benefit of 27 months compared to EBRT alone (48.6 vs. 21.6 months, HR: $0.475,95 \%$ CI: $0.255-0.883$, p $=0.019)$.

The largest dataset to date aimed at studying PCI for extra-pulmonary small cell carcinoma was compiled by Naidoo, et al. and studied 280 patients, of whom 186 patients had extensive-stage disease, 65 patients had limited stage disease, and 29 patients had an unknown stage [10]. Of the 280 patients analysed, 18 patients (6.4\%) developed brain metastases. Only $2.5 \%$ of the patient cohort presented with brain metastases at initial diagnosis, which is a significantly lower incidence than is seen for SCLC. Overall, the data published by Naidoo, et al. does not support the use of PCI due to low incidence of brain metastases in SCCC.

\section{Conclusion}

Multicentric clinical trials are needed to try to find an unambiguous and effective treatment for small cell neuroendocrine carcinoma of the cervix to improve patient survival.

\section{References}

1. Lee SW, Lim KT, Bae DS, Park SY, Kim YT, et al. (2015) A multicenter study of the importance of systemic chemotherapy for patients with small-cell neuroendocrine carcinoma of the uterine cervix. Gynecol Obstet Investig 79(3): 172-178.

2. Korcum AF, Aksu G, Bozcuk H, Pesterli E, Simsek T (2008) Small cell carcinoma of the cervix: a case report. Arch Gynecol Obstet 277(4): 367-370.

3. Berniker AV, Abdulrahman AA, Teytelboym OM, Galindo LM, Mackey JE (2015) Extrapulmonary small cell carcinoma: imaging features with radiologicpathologic correlation. Radiographics 35(1): 152-163.

4. Gardner GJ, Lagunes DR, Gehrig PA (2011) Neuroendocrine tumors of the gynecologic tract: a society of gynecologic oncology (SGO) clinical document. Gynecol Oncol 122(1): 190-198.

5. Zheng X, Liu D, Fallon JT, Zhong M (2015) Distinct genetic alterations in small cell carcinoma from different anatomic sites. Exp Hematol Oncol 4: 2.

6. Chen TC, Huang HT, Wang TY, Yang LY, Chen CH, et al. (2015) Primary surgery versus primary radiation therapy for FIGO stages I-II small cell carcinoma of the uterine cervix: a retrospective Taiwanese gynecologic oncology group study. Gynecol Oncol 137(3): 468-473.

7. Wang KL, Chang TC, Jung SM, Chen CH, Cheng YM, et al. (2012) Primary treatment and prognostic factors of small cell neuroendocrine carcinoma of the uterine cervix: a Taiwanese Gynecologic Oncology Group study. Eur J Cancer 48(10): 1484-1494.

8. Frumovitz M (2016) Small- and large-cell neuroendocrine cervical cancer. Oncology (Williston Park) 30(1): 70-78.

9. Robin TP, Amini A, Schefter TE, Behbakht K, Fisher CM (2016) Brachytherapy should not be omitted when treating locally advanced neuroendocrine 


\section{International Journal of Nuclear Medicine \& Radioactive Substances}

cervical cancer with definitive chemoradiation therapy. Brachytherapy 15(6): 845-850.

10. Naidoo J, Teo MY, Deady S, Comber H, Calvert P (2013) Should patients with extrapulmonary small- cell carcinoma receive prophylactic cranial irradiation?. J Thoracic Oncol 8(9): 1215-1221. 